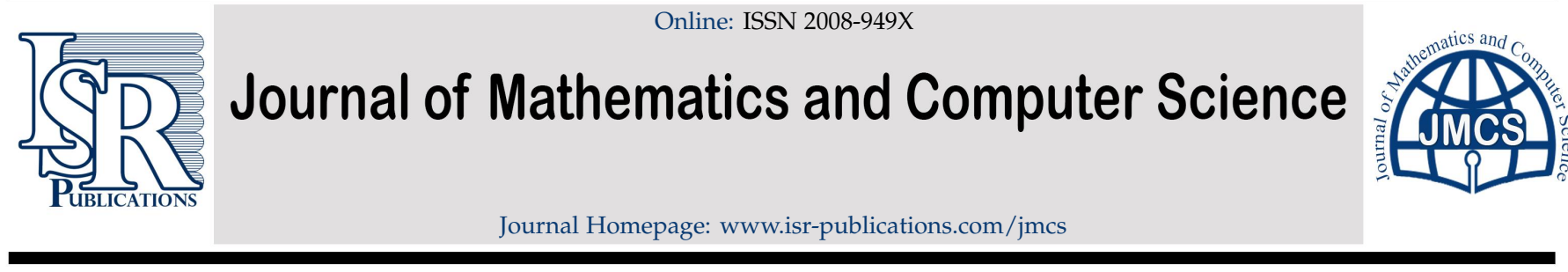

\title{
Analytical solution of systems of Volterra integro- differential equations using modified differential transform method
}

Shadi Al-Ahmad ${ }^{\mathrm{a}}$, Ibrahim Mohammed Sulaiman ${ }^{\mathrm{a}, *}$, Mohamad Arif Awang Nawi ${ }^{\mathrm{b}}$, Mustafa Mamat ${ }^{\mathrm{a}}$, Muhammad Zaini Ahmad'

${ }^{a}$ Faculty of Informatics and Computing, Universiti Sultan Zainal Abidin, Besut Campus, 22200, Kuala Terengganu, Malaysia.

${ }^{b}$ School of Dental Sciences, Health Campus, Universiti Sains Malaysia (USM), 16150 Kubang Kerian, Kota Bharu, Kelantan, Malaysia.

${ }^{c}$ Institute of Engineering Mathematics, Universiti Malaysia Perlis, 02600, Arau Perlis, Malaysia.

\begin{abstract}
In this paper, a hybrid technique based on the combining of differential transform approach, the Laplace transformation, and Padé approximant is successfully implemented for solving Volterra Integro differential (VID) equations subject to appropriate conditions. The proposed method known as modified differential transform method (MDTM) enlarges the domain of convergence with less computational time. One of the interesting features of this algorithm is the ability to produce an analytic approximate solution in convergent power series form with few numbers of computable terms. Preliminary results on some test problems show that the new algorithm is efficient and promising for Volterra integro-differential equations and can be applied as alternatives for the integral and integro-differential (IID) equation that have no analytical solution.
\end{abstract}

Keywords: Numerical approximation, differential transform method, Volterra integro-differential equations.

2020 MSC: 30C45, M35F55, 26A33.

(C)2022 All rights reserved.

\section{Introduction}

Nonlinear phenomena such as those resulting from the interaction between intense light beams with dielectric media are often traced to fields science and engineering. These phenomena which appear in many real-life applications including chemical kinetics, fluid dynamics, mathematical biology, solid-state physics, are often modeled by partial differential equations (PDEs) [20]. The solution of these PDEs are obtained by different numerical or analytical methods. One of the earlier approach for obtaining the solution partial differential equation is using the differential transform technique (DTM) introduce by Zhou [37] and gives an analytical Taylor solution in the form of series. This method is a recursive method that produce an analytical solution in polynomial form and gives the exact values of the nth derivative in a fast manner for both known and unknown boundary conditions via the concept of Taylors series $[12,19,33]$. However, one of the many disadvantage of this method is that the truncated series obtained

\footnotetext{
${ }^{*}$ Corresponding author

Email address: sulaimanib@unisza.edu.my (Ibrahim Mohammed Sulaiman)

doi: $10.22436 /$ jmcs.026.01.01
}

Received: 2021-06-28 Revised: 2021-07-14 Accepted: 2021-08-10 
which is an accurate approximation of exact solution is often in very small region [31]. The setback of this method has led to numerous modifications of the differential transform method.

Its is worthy to note that various real-life problems can be modelled under differential sense to produce the differential equation [10]. Recently, several studies have been done on numerical methods for solving various types of differential equation including the two-dimensional nonlinear Klein-Gordon equations [13], partial algebraic-differential equations [14], IID equations [7], pantograph-type linear and nonlinear VID equations [32]. The concept of integral and integro-differential problems plays an important part in indutrial and engineering applications. These problems have been considered by numerous researchers. For instance, [15, 34], considered Volterra integral equations using the Adomian decomposition technique (ADM) while [12] employ the Galerkin technique for linear-integro DE. Rationalized Haar functions technique was employed for obtaining solution of linear-integro differential equation by [30, 36], [16] extended the He's homotopy perturbation approach (HPM) to solve system of integro-differential equations. Their approach relies on the permutation of pertubation procedure in topological scheme that are first presented by [21-24]. Also, [35] employed the variational iteration technique (VIM) for solving intrgro-differential equations. Using the general Lagrange multiplier, the authors develop a correction function whose identification are obtained using the variational theory. A study comparing the methods of DTM, ADM, and VIM for solving PDEs was presented by Bildik et al. [17, 18]. Also, review of different methods for solving different type of differential equations is given by $[2-4,26,28,29]$.

Motivated by the trend of current literature, this study proposed an improve solution scheme for Volterra integro-differential system of equations. The new method would modify the series solution via the following procedure. The algorithm begins with the Laplace transform approach to DTM obtained truncated series. To obtain meromorphic function from the transformed series, we used the approximants of Padé and lastly employing the inverse Laplace transformation to get the desired analytical solution. Result of numerical experiment on some test problems are given to demonstrate the effectiveness of the proposed algorithm compared to the truncated series solution of DTM.

The remaining part of the paper is structured as follow. A brief overview of recent literature followed by some preliminary results is presented in Section 2. In Section 3, we present two applications of Volterra integro-differential systems of equations to demonstrate efficiency and robustness of the new algorithm. Finally, the conclusion and discussion for further reference is given in Section 4.

\section{Preliminaries}

Preliminary discussion of different literature on this topic is presented in this section.

\subsection{Differential transform approach}

Definition 2.1 ([6]). Given an analytical function $f(x)$ at the point $x_{0}$ in domain of interest (DOI), we have

$$
F(k)=\frac{f^{(k)}\left(x_{0}\right)}{k !}
$$

whose inverse is

$$
f(x)=\sum_{k=0}^{\infty} F(k)\left(x-x_{0}\right)^{k} .
$$

Equation (2.2) is known as the inverse of (2.1). Combining (2.1), (2.2), will produce beginequation

$$
f(x)=\sum_{k=0}^{\infty} \frac{f^{(k)}\left(x_{0}\right)}{k !}\left(x-x_{0}\right)^{k} .
$$

If $U(k), G(k)$, and $H(k)$ denote the main operators of the differential transform $u(x), g(x)$, and $h(x)$ respectively, it would implies that the operators in Table1 represent the operations of the DTM. 
Table 1: Differential transform operations

\begin{tabular}{|l|l|}
\hline Function & Differential Transform \\
\hline$u(x)=g(x)+h(x)$ & $U(k)=G(k)+H(k)$ \\
\hline$u(x)=c g(x)$ & $U(k)=c G(k)$ \\
\hline$u(x)=\frac{d^{n} g(x)}{d x^{n}}$ & $U(k)=\frac{(k+n) !}{k !} G(k+n)$ \\
\hline$u(x)=g(x) h(x)$ & $U(k)=\sum_{i=0}^{k} G(i) H(k-i)$ \\
\hline$u(x)=x^{n}$ & $U(k)=\delta(k-n)$ \\
\hline$u(x)=\exp (c x)$ & $U(k)=\frac{c^{k}}{k !}$ \\
\hline$u(x)=\cos (\omega x)$ & $U(k)=\frac{\omega^{k}}{k !} \cos \left(\frac{k \pi}{2}\right)$ \\
\hline$u(x)=\sin (\omega x)$ & $U(k)=\frac{w^{k}}{k !} \sin \left(\frac{k \pi}{2}\right)$ \\
\hline
\end{tabular}

Theorem 2.2 ([9]). If $f(y)=y^{m}$, we have

$$
F(k)= \begin{cases}(Y(0))^{m}, & k=0, \\ \frac{1}{Y(0)} \sum_{r=1}^{k}\left(\frac{(m+1) r-k}{k}\right) Y(r) F(k-r), & k \geqslant 1 .\end{cases}
$$

Theorem 2.3 ([9]). If $f(y)=e^{a y}$, then

$$
F(k)= \begin{cases}e^{a Y(0)} & k=0 \\ a \sum_{r=0}^{k-1}\left(\frac{r+1}{k}\right) Y(r+1) F(k-1-r), & k \geqslant 1\end{cases}
$$

Theorem 2.4 ([9]). If $\sin (\alpha y)$ and $\cos (\alpha y)$ represent $f(y)$ and $g(y)$, respectively, then

$$
F(k)=\left\{\begin{array}{ll}
\sin (\alpha Y(0)), & k=0, \\
\alpha \sum_{r=0}^{k-1}\left(\frac{k-r}{k}\right) G(r) Y(k-r), & k \geqslant 1,
\end{array} \quad \text { and } \quad G(k)= \begin{cases}\cos (\alpha Y(0)), & k=0, \\
-\alpha \sum_{r=0}^{k-1}\left(\frac{k-r}{k}\right) F(r) Y(k-r), & k \geqslant 1 .\end{cases}\right.
$$

Theorem 2.5 ([33]). Suppose the DT of the functions $\mathrm{u}(\mathrm{x})$ and $\mathrm{g}(\mathrm{x})$ are $\mathrm{U}(\mathrm{k})$ and $\mathrm{G}(\mathrm{k})$, then:

1. for $f(x)=\int_{x_{0}}^{x} u(t) d t$, we have $F(k)=\frac{u(k-1)}{k}, F(0)=0$;

2. for $\mathrm{f}(\mathrm{x})=\int_{\mathrm{x}_{0}}^{\mathrm{x}} \mathrm{g}(\mathrm{t}) \mathrm{u}(\mathrm{t}) \mathrm{dt}$, we have $\mathrm{F}(\mathrm{k})=\sum_{\mathrm{l}=0}^{\mathrm{k}-1} \mathrm{G}(\mathrm{l}) \frac{\mathrm{u}(\mathrm{k}-\mathrm{l}-1)}{\mathrm{k}}, \mathrm{F}(0)=0$;

3. for $f(x)=g(x) \int_{x_{0}}^{x} u(t) d t$, we have $F(k)=\sum_{l=0}^{k-1} G(l) \frac{u(k-l-1)}{k-l}, F(0)=0$.

By means of the DT, the differential equation in DOI is converted to algebraic equation in the K-domain and function $f(t)$ is computed using the Taylor series finite-term expansion in addition to a remainder, as

$$
f(t)=\sum_{k=0}^{N} F(k) \frac{\left(t-t_{0}\right)^{k}}{k !}+R_{N+1}(t) .
$$

In a small region, the series solution in (2.3) converges rapidly, however, the convergence results are often time consuming in the larger region and hence, their truncations yield inexact results. For recent studies on this subject, see $[5,6]$.

\subsection{Padé approximation $[8,9]$}

The Padé approximant for Taylor series expansion of $y(x)$ of order $[L / M]$ is largely denoted as $[L / M]$ PA to $y(x)$ and defined below

$$
\left[\frac{\mathrm{L}}{\mathrm{M}}\right]=\frac{\mathrm{P}_{\mathrm{L}}(\mathrm{x})}{\mathrm{Q}_{\mathrm{M}}(\mathrm{x})} .
$$


The polynomials $\mathrm{P}_{\mathrm{L}}(\mathrm{x})$ and $\mathrm{Q}_{\mathrm{M}}(\mathrm{x})$ are of degree at most $\mathrm{L}$ and $M$. The series

$$
\begin{gathered}
y(x)=\sum_{i=1}^{\infty} a_{i} x^{i}, \\
y(x)-\frac{P_{L}(x)}{Q_{M}(x)}=O\left(x^{L+M+1}\right),
\end{gathered}
$$

are used to obtain the parameters of $P_{L}(x)$ and $Q_{M}(x)$. It is obvious that $[L / M]$ will remain unchanged when the denominator and numerator is multiply by certain constant, thus the normalization condition

$$
\mathrm{Q}_{M}(0)=1
$$

is imposed on the equation and the polynomials are said to have no common factors. Rewrite coefficients of the functions $\mathrm{P}_{\mathrm{L}}(\mathrm{x})$ and $\mathrm{Q}_{\mathrm{M}}(\mathrm{x})$ as

$$
\left\{\begin{array}{l}
P_{L}(x)=p_{0}+p_{1} x+p_{2} x^{2}+\cdots+p_{L} x^{L} \\
Q_{M}(x)=q_{0}+q_{1} x+q_{2} x^{2}+\cdots+q_{M} x^{M}
\end{array}\right.
$$

Using (2.5) and (2.6), the coefficient equations are linearized after multiplying (2.4) by $\mathrm{Q}_{M}(\mathrm{x})$. Eq (2.4) is presented as

$$
\begin{aligned}
& \left\{\begin{array}{l}
a_{L+1}+a_{L} q_{1}+\cdots+a_{L-M+1} q_{M}=0 \\
a_{L+2}+a_{L+1} q_{1}+\cdots+a_{L-M+2} q_{M}=0 \\
\vdots \\
a_{L+M}+a_{L+M-1} q_{1}+\cdots+a_{L} q_{M}=0
\end{array}\right. \\
& \left\{\begin{array}{l}
a_{0}=p_{0} \\
a_{0}+a_{0} q_{1}=p_{1} \\
a_{2}+a_{1} q_{1}+a_{0} q_{2}=p_{2} \\
\vdots \\
a_{L}+a_{L-1} q_{1}+\cdots+a_{0} q_{L}=p_{L}
\end{array}\right.
\end{aligned}
$$

Equation (2.7) is a set of linear equation. To compute for the solution of (2.7), we solve to obtain the values q's that are not known to make equation (2.8) inevitably give the unambiguous formulation for the undefined $p^{\prime} s$, and result would be complete.

However, the case of non-singularity of equations (2.7) and (2.8) would be directly solved to get (2.9).

$$
\left[\frac{L}{M}\right]=\frac{\operatorname{det}\left[\begin{array}{cccc}
a_{L-M+1} & a_{L-M+2} & \cdots & a_{L+1} \\
\vdots & \vdots & \ddots & \vdots \\
a_{L} & a_{L+1} & \cdots & a_{L+M} \\
\sum_{j=M}^{L} a_{j-M} x^{j} & \sum_{j=M-1}^{L} a_{j-M+1} x^{j} & \cdots & \sum_{j=0}^{L} a_{j} x^{j}
\end{array}\right]}{\operatorname{det}\left[\begin{array}{cccc}
a_{L-M+1} & a_{L-M+2} & \cdots & a_{L+1} \\
\vdots & \vdots & \ddots & \vdots \\
a_{L} & a_{L+1} & \cdots & a_{L+M} \\
x^{M} & x^{M-1} & \cdots & 1
\end{array}\right]} .
$$

In the case where the index sum of the lower index exceed that of the upper and (2.9) holds, then, we replaced the sum with zero. In this cases, MATLAB symbolic software, is used to obtain Padé approximants diagonal elements of various orders which may include: [2/2], [4/4], or [6/6].

The Padé approximant has some poles that are not available in the original function. Expanding Padé $[M / N]$ in the series of Taylor's equation and computing its parameters is an easy computational task to that can be achieved by matching with the above.

The MDTM procedure for the proposed system of equations is given via the following algorithm. 
Algorithm 2.6. MDTM for system of VID equations.

Step 1: Having $y_{j}{ }^{(n)}=g_{j}(t)+\int_{0}^{t} K_{j}(x, t) d x, y_{j}(0)=\alpha_{j}, y_{j}{ }^{\prime}(0)=\beta_{j}, \ldots, y_{j}{ }^{(n-1)}(0)=\gamma_{j} 0 \leqslant t \leqslant 1$, the exact solution $y_{j}(t), j=1,2,3, \ldots$

Step 2: Fix $M=2$.

Step 3: Calculate $Y_{j}(k)=\frac{1}{k !}\left[\frac{d^{k}}{d x^{k}} y(t)\right]_{t=0}, k=0,1,2, \ldots, N, j=1,2,3, \ldots$

Step 4: Expand $f_{j}(t)=\sum_{k=0}^{N} Y_{i}(k) t^{k}, j=1,2,3, \ldots$

Step 5: Obtain $\mathcal{L}\left(f_{j}(t)\right)=\mathcal{L}\left(\sum_{k=0}^{N} Y_{i}(k) t^{k}\right), j=1,2,3, \ldots$

Step 6: Replace $1 / \mathrm{s}=z$ in Step 5 .

Step 7: Compute $\left[\frac{M}{M}\right]\left(\mathcal{L}\left(f_{j}(t)\right)\right), j=1,2,3, \ldots$

Step 8: Replace $z=\frac{1}{s}$ in Step 7 .

Step 9: Calculate $\mathcal{L}^{-1}\left(\left[\frac{M}{M}\right]\left(\mathcal{L}\left(f_{j}(t)\right)\right)\right), j=1,2,3, \ldots$

Step 10: If $\max _{0 \leqslant t_{i} \leqslant 1}\left|y_{j}\left(t_{i}\right)-\left(\mathcal{L}^{-1}\left(\left[\frac{M}{M}\right]\left(\mathcal{L}\left(f_{j}(t)\right)\right)\right)\right)\left(t_{i}\right)\right| \leqslant 10^{-6}, \forall j=1,2,3, \ldots$, terminate, otherwise, return to step 7 with $M=M+1$.

\section{Numerical results}

Problem 3.1. Given the Volterra type integro-differential equation system [11]

$$
\left\{\begin{array}{l}
u_{1}^{\prime}(t)=1+t+t^{2}-u_{2}(t)-\int_{0}^{t}\left[u_{1}(x)+u_{2}(x)\right] d x \\
u_{2}^{\prime}(t)=-1-t+u_{1}(t)-\int_{0}^{t}\left[u_{1}(x)-u_{2}(x)\right] d x
\end{array}\right.
$$

where $\mathfrak{u}_{1}(0)=1, \mathfrak{u}_{2}(0)=-1$, and $u(t)=\left[u_{1}(t), u_{2}(t)\right]^{\top}=\left[t+e^{t}, t-e^{t}\right]^{\top}$ is the exact solution, by Theorem 2.5 and using operations from Table 1, we have

$$
\left\{\begin{array}{l}
\mathrm{U}_{1}(\mathrm{k}+1)=\frac{1}{\mathrm{k}+1}\left[\delta(\mathrm{k})+\delta(\mathrm{k}-1)+\delta(\mathrm{k}-2)-\mathrm{U}_{2}(\mathrm{k})-\frac{1}{\mathrm{k}}\left[\mathrm{U}_{1}(\mathrm{k}-1)+\mathrm{U}_{2}(\mathrm{k}-1)\right]\right], \\
\mathrm{U}_{2}(\mathrm{k}+1)=\frac{1}{\mathrm{k}+1}\left[-\delta(\mathrm{k})-\delta(\mathrm{k}-1)+\mathrm{U}_{1}(\mathrm{k})-\frac{1}{\mathrm{k}}\left[\mathrm{U}_{1}(\mathrm{k}-1)-\mathrm{U}_{2}(\mathrm{k}-1)\right]\right],
\end{array}\right.
$$

and transforming the initial condition to get

$$
\left\{\begin{array}{l}
\mathrm{u}_{1}(0)=1 \\
\mathrm{U}_{2}(0)=-1
\end{array}\right.
$$

We substitute (3.3) in (3.2) to obtain the results of $U_{1}(k)$ and $U_{2}(k)$ defined in Table 2.

Table 2: The values of $U_{1}(k)$ and $U_{2}(k)$ for Problem 3.1.

\begin{tabular}{ccccccc}
\hline $\mathrm{k}$ & 0 & 1 & 2 & 3 & 4 & 5 \\
\hline $\mathrm{U}_{1}(\mathrm{k})$ & 1 & 2 & $\frac{1}{2}$ & $\frac{1}{6}$ & $\frac{1}{24}$ & $\frac{1}{120}$ \\
\hline $\mathrm{U}_{2}(\mathrm{k})$ & -1 & 0 & $-\frac{1}{2}$ & $-\frac{1}{6}$ & $-\frac{1}{24}$ & $-\frac{1}{120}$ \\
\hline
\end{tabular}

An approximate solution of equation (3.1) is obtained using the rule (2.2) of the inverse transformation

$$
\left\{\begin{array}{l}
f_{1}(t)=1+2 t+\frac{t^{2}}{2}+\frac{t^{3}}{6}+\frac{t^{4}}{24}+\frac{t^{5}}{120}+\cdots, \\
f_{2}(t)=-1-\frac{t^{2}}{2}-\frac{t^{3}}{6}-\frac{t^{4}}{24}-\frac{t^{5}}{120}-\cdots,
\end{array}\right.
$$

which gives the solution (3.1) in the limit of infinitely many terms (LIMT). The proposed method is implemented via the Laplace transform to the (3.4), which gives

$$
\left\{\begin{array}{l}
\mathcal{L}\left(f_{1}(t)\right)=\frac{1}{s}+\frac{2}{s^{2}}+\frac{1}{s^{3}}+\frac{1}{s^{4}}+\frac{1}{s^{5}}+\frac{1}{s^{6}}+\cdots \\
\mathcal{L}\left(f_{2}(t)\right)=-\frac{1}{s}-\frac{1}{s^{3}}-\frac{1}{s^{4}}-\frac{1}{s^{5}}-\frac{1}{s^{6}}+\cdots
\end{array}\right.
$$


To ease the process, let $\mathrm{s}=\frac{1}{z}$, then

$$
\left\{\begin{array}{l}
\mathcal{L}\left(f_{1}(t)\right)=z+2 z^{2}+z^{3}+z^{4}+z^{5}+z^{6}+\cdots \\
\mathcal{L}\left(f_{2}(t)\right)=-z-z^{3}-z^{4}-z^{5}-z^{6}+\cdots
\end{array}\right.
$$

Table 3 shows how Algorithm 2.6 works indicating the max absolute error for each choice of Padé approximant.

Table 3: Algorithm 2.6 works for Problem 3.1.

\begin{tabular}{cccc}
\hline$\left[\frac{M}{M}\right]$ & Padé approximant for $\mathcal{L}\left(f_{1}(t)\right)$ & $\mathcal{L}^{-1}\left(\left[\frac{M}{M}\right]\left(\mathcal{L}\left(f_{1}(t)\right)\right)\right.$ & The max absolute Error \\
\hline$\left[\frac{2}{2}\right]$ & $-\frac{5 z^{2}+3 z}{z^{2}+z-3}$ & $e^{\frac{t}{6}}\left(\cosh \left(\frac{\sqrt{13} t}{6}\right)+\frac{11 \sqrt{13}}{13} \sinh \left(\frac{\sqrt{13} t}{6}\right)\right)$ & $1.857164054955351 E-2$ \\
\hline$\left[\frac{3}{3}\right]$ & $\frac{z^{3}-z^{2}-z}{z-1}$ & $t+e^{t}$ & 0 \\
\hline$\left[\frac{M}{M}\right]$ & Padé approximant for $\mathcal{L}\left(f_{2}(t)\right)$ & $\mathcal{L}^{-1}\left(\left[\frac{M}{M}\right]\left(\mathcal{L}\left(f_{2}(t)\right)\right)\right.$ & The max absolute Error \\
\hline$\left[\frac{2}{2}\right]$ & $-\frac{z^{2}-z}{z^{2}+z-1}$ & $-e^{\frac{t}{2}}\left(\cosh \left(\frac{\sqrt{5} t}{2}\right)-\frac{\sqrt{5}}{5} \sinh \left(\frac{\sqrt{5} t}{2}\right)\right)$ & 6.564116785383356E-2 \\
\hline$\left[\frac{3}{3}\right]$ & $\frac{z^{3}-z^{2}+z}{z-1}$ & $t-e^{t}$ & 0 \\
\hline
\end{tabular}

For $\left[\frac{3}{3}\right]$, we have

$$
\left[\frac{3}{3}\right]=\left\{\begin{array}{l}
\mathcal{L}\left(f_{1}(t)\right)=\frac{z^{3}-z^{2}-z}{z-1} \\
\mathcal{L}\left(f_{2}(t)\right)=\frac{z^{3}-z^{2}+z}{z-1} .
\end{array}\right.
$$

Recalling $z=\frac{1}{s}$ to obtain $\left[\frac{3}{3}\right]$ in respect of $s$,

$$
\left[\frac{3}{3}\right]=\left\{\begin{array}{l}
\mathcal{L}\left(f_{1}(t)\right)=\frac{s^{2}+s-1}{s^{3}-s^{2}} \\
\mathcal{L}\left(f_{2}(t)\right)=\frac{-s^{2}+s-1}{s^{3}-s^{2}}
\end{array}\right.
$$

For obtaining the modified approximation, the study employs the inverse to Padé $\left[\frac{3}{3}\right]$ approximant

$$
\mathcal{L}^{-1}\left(\left[\frac{3}{3}\right]\left(\mathcal{L}\left(f_{j}(t)\right)\right)\right)=\left\{\begin{array}{l}
t+e^{t} \\
t-e^{t}
\end{array}\right.
$$

which is in good agreement with the exact solution.

Figure 1 presents the plot of exact solution $y_{j}(t), j=1,2$ for Problem 3.1 with comparison to that of DTM solution which all result are in excellent agreement.
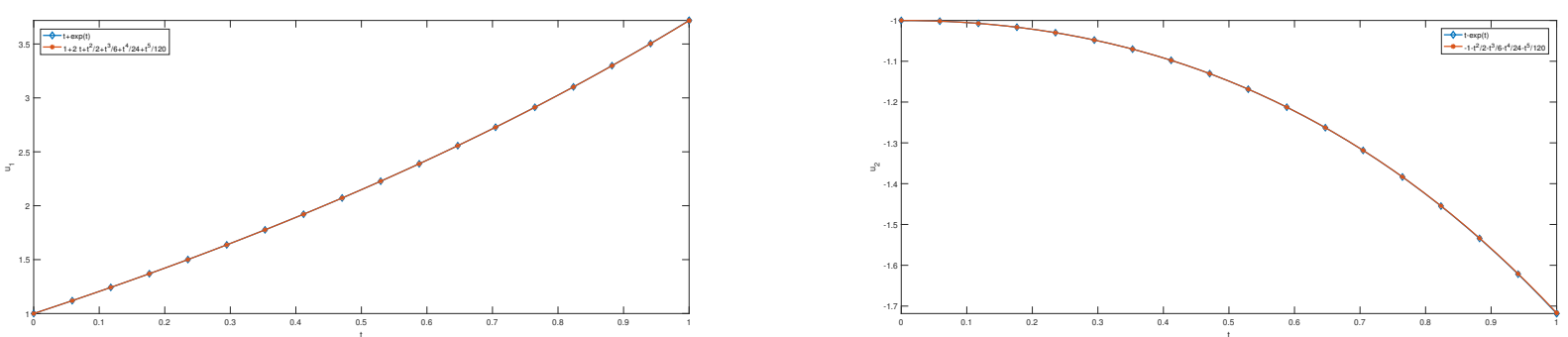

Figure 1: Performance Comparison of DTM and MDTM for Problem 3.1.

Problem 3.2. Consider the system of VID equation [1]

$$
\left\{\begin{array}{l}
u_{1}{ }^{\prime \prime}(t)=-\sin (t)-t^{2}-1+\int_{0}^{t}\left[u_{1}(x)+u_{2}(x)\right] d x \\
u_{2}{ }^{\prime \prime}(t)=1-\cos (t)-2 \sin (t)+\int_{0}^{t}\left[u_{1}(x)-u_{2}(x)\right] d x,
\end{array}\right.
$$


where $u_{1}(0)=1, u_{1}^{\prime}(0)=1, u_{2}(0)=0, u_{2}{ }^{\prime}(0)=2$, and $u(t)=\left[u_{1}(t), u_{2}(t)\right]^{\top}=[t+\cos (t), t+\sin (t)]^{\top}$ is the exact solution. By Theorem 2.5 and using operations from Table 1, we have

$$
\left\{\begin{array}{l}
\mathrm{U}_{1}(\mathrm{k}+2)=\frac{1}{(\mathrm{k}+1)(\mathrm{k}+2)}\left[-\frac{1}{\mathrm{k} !} \sin \left(\frac{\mathrm{k} \pi}{2}\right)-\delta(\mathrm{k}-2)-\delta(\mathrm{k})+\frac{1}{\mathrm{k}}\left[\mathrm{U}_{1}(\mathrm{k}-1)+\mathrm{U}_{2}(\mathrm{k}-1)\right]\right] \\
\mathrm{U}_{2}(\mathrm{k}+2)=\frac{1}{(k+1)(\mathrm{k}+2)}\left[\delta(\mathrm{k})-\frac{1}{\mathrm{k} !} \cos \left(\frac{\mathrm{k} \pi}{2}\right)-\frac{2}{\mathrm{k} !} \sin \left(\frac{\mathrm{k} \pi}{2}\right)+\frac{1}{\mathrm{k}}\left[\mathrm{U}_{1}(\mathrm{k}-1)+\mathrm{U}_{2}(\mathrm{k}-1)\right]\right]
\end{array}\right.
$$

Transform the initial condition to get

$$
\left\{\begin{array}{l}
\mathrm{u}_{1}(0)=1, \mathrm{u}_{1}(1)=1 \\
\mathrm{U}_{2}(0)=0, \mathrm{U}_{2}(1)=2
\end{array}\right.
$$

We substitute (3.7) in (3.6) to get the results of $U_{1}(k)$ and $U_{2}(k)$ given in Table 4 .

Table 4: The values of $\mathrm{U}_{1}(\mathrm{k})$ and $\mathrm{U}_{2}(\mathrm{k})$ for Problem 3.2.

\begin{tabular}{cccccccccc}
\hline $\mathrm{k}$ & 0 & 1 & 2 & 3 & 4 & 5 & 6 & 7 & 8 \\
\hline $\mathrm{U}_{1}(\mathrm{k})$ & 1 & 1 & $-\frac{1}{2}$ & 0 & $\frac{1}{24}$ & 0 & $-\frac{1}{720}$ & 0 & $\frac{1}{40320}$ \\
\hline $\mathrm{U}_{2}(\mathrm{k})$ & 0 & 2 & 0 & $-\frac{1}{6}$ & 0 & $\frac{1}{120}$ & 0 & $-\frac{1}{5040}$ & 0 \\
\hline
\end{tabular}

To derive the solution of (3.5), we apply rule (2.2) of the inverse transformation as follows:

$$
\left\{\begin{array}{l}
f_{1}(t)=1+t-\frac{t^{2}}{2}+\frac{t^{4}}{24}-\frac{t^{6}}{720}+\cdots, \\
f_{2}(t)=2 t-\frac{t^{3}}{6}+\frac{t^{5}}{120}-\frac{t^{7}}{5040}+\cdots,
\end{array}\right.
$$

and this gives which the exact solution of (3.5) infinitely many terms limit. The proposed algorithm is implemented using the Laplace transform on (3.8), to obtain

$$
\left\{\begin{array}{l}
\mathcal{L}\left(f_{1}(t)\right)=\frac{1}{s}+\frac{1}{s^{2}}-\frac{1}{s^{3}}+\frac{1}{s^{5}}-\frac{1}{s^{7}}+\cdots \\
\mathcal{L}\left(f_{2}(t)\right)=\frac{2}{s^{2}}-\frac{1}{s^{4}}+\frac{1}{s^{6}}-\frac{1}{s^{8}}+\cdots
\end{array}\right.
$$

To simplify the process, we let $s=\frac{1}{z}$, then

$$
\left\{\begin{array}{l}
\mathcal{L}\left(f_{1}(t)\right)=z+z^{2}-z^{3}+z^{5}-z^{7}+\cdots \\
\mathcal{L}\left(f_{2}(t)\right)=2 z^{2}-z^{4}+z^{6}-z^{8}+\cdots
\end{array}\right.
$$

The Padé approximants $\left[\frac{4}{4}\right]$ gives

$$
\left[\begin{array}{l}
\frac{4}{4}
\end{array}\right]=\left\{\begin{array}{l}
\mathcal{L}\left(f_{1}(t)\right)=\frac{z^{4}+z^{2}+z}{z^{2}+1} \\
\mathcal{L}\left(f_{2}(t)\right)=\frac{z^{4}+2 z^{2}}{z^{2}+1} .
\end{array}\right.
$$

Recalling $z=\frac{1}{s}$ to get $\left[\frac{4}{4}\right]$ in respect of $s$,

$$
\left[\begin{array}{l}
\frac{4}{4}
\end{array}\right]=\left\{\begin{array}{l}
\mathcal{L}\left(f_{1}(t)\right)=\frac{s^{3}+s^{2}+1}{s^{4}+s^{2}} \\
\mathcal{L}\left(f_{2}(t)\right)=\frac{2 s^{2}+1}{s^{4}+s^{2}}
\end{array}\right.
$$

To compute for the modified approximation, we use the inverse of the Padé $\left[\frac{4}{4}\right]$ approximant as follows:

$$
\mathcal{L}^{-1}\left(\left[\frac{4}{4}\right]\left(\mathcal{L}\left(f_{j}(t)\right)\right)\right)=\left\{\begin{array}{l}
t+\cos (t), \\
t+\sin (t),
\end{array}\right.
$$

which agrees with the exact solution. Figure 2 shows the graph of the exact solution $y_{j}(t), j=1,2$ compared with DTM solution for Problem 3.2. 

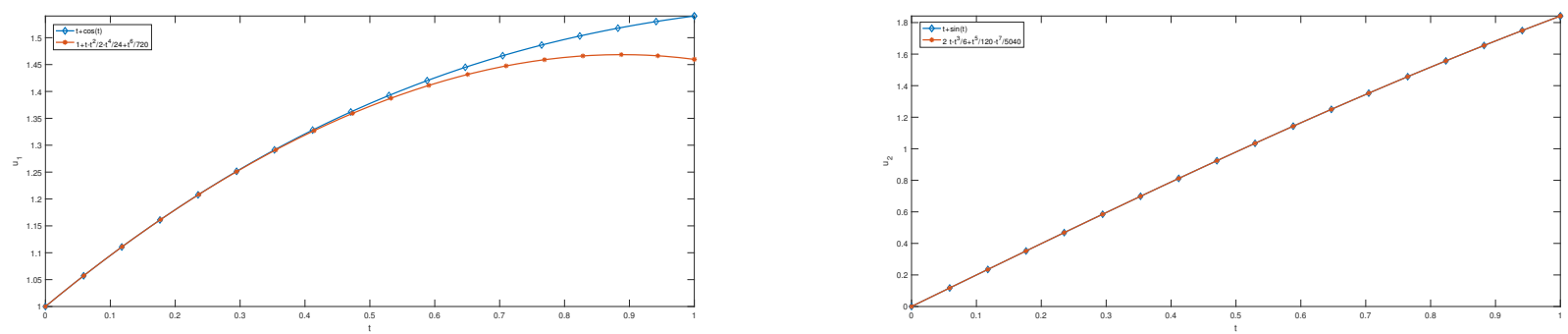

Figure 2: Performance Comparison of DTM and MDTM for Problem 3.2.

The results presented in Figure 2 show that all methods are in excellent agreement with result of the exact solutions.

\section{Conclusion and discussion}

This paper presents the MDTM method derived by combining of the DTM, Padé approximant, and Laplace transforms for solving system of VID equations. The method begins by obtaining the PDEs in the form of convergent series via DTM. Next, Laplace transform procedure is employed to the truncated series of DTM solution to widen the convergence domain. Lastly, the meromorphic function is obtained from the series via Padé approximants whose solution is obtained using the inverse Laplace transform. By solving two system of VID equations, the proposed algorithm is seen as a tool with great potential. In addition, we successfully obtained the exact solutions by illustrating the efficiency of MDTM. The result of the problems indicates that MDTM greatly improves the convergence rate of DTM's truncated series solution with true analytic solution. Therefore, the proposed algorithm can be used as alternatives for systems of integral and integro-differential equation that have no analytical solution.

\section{Acknowledgment}

The authors are grateful to the reviewers for their comments and also Universiti Sultan Zainal Abidin, Malaysia. This work was supported by School of Dental Sciences, Health Campus, Universiti Sains Malaysia under the the Short Term Grant Number: 304/PPSG/6315410.

\section{References}

[1] P. Agarwal, M. Akbar, R. Nawaz, M. Jleli, Solutions of system of Volterra integro-differential equations using optimal homotopy asymptotic method, Math. Methods Appl. Sci., 44 (2020), 2671-2681. 3.2

[2] R. AlAhmad, Laplace transform of the product of two functions, Ital. J. Pure Appl. Math., 2020 (2020), 800-804. 1

[3] R. AlAhmad, M. Al-Jararha, On solving some classes of second order ODEs, Italian J. Pure Appl. Math., 45 (2021), 673-688.

[4] R. AlAhmad, M. Al-Jararha, H. AlMefleh, Exactness of second order ordinary differential equations and integrating factors, Jordan J. Math. Stat., 11 (2015), 155-167. 1

[5] S. Al-Ahmad, M. Mamat, R. Al-Ahmad, Finding Differential Transform Using Difference Equations, IAENG Int. J. Appl. Math., 50 (2020), 127-132. 2.1

[6] S. Al-Ahmad, M. Mamat, R. Al-Ahmad, I. M. Sulaiman, P. L. Ghazali, M. A. Mohamed, On New Properties of Differential Transform via Difference Equations, Int. J. Eng. Tech., 7 (2018), 321-324. 2.1, 2.1

[7] S. Al-Ahmad, I. M. Sulaiman, M. Mamat, An Efficient Modification of Differential Transform Method for Solving Integral and Integro-Differential Equations, Aust. J. Math. Anal. Appl., 17 (2020), 15 pages. 1

[8] S. Al-Ahmad, I. M. Sulaiman, M. Mamat, P. L. Ghazali, Modified differential transform scheme for solving systems of first order ordinary differential equations, J. Math. Comput. Sci., 22 (2021), 73-84. 2.2

[9] S. Al-Ahmad, I. M. Sulaiman, M. Mamat, K. Kamfa, Solutions of classes of differential equations using modified differential transform method, J. Math. Comput. Sci., 10 (2020), 2360-2382. 2.2, 2.3, 2.4, 2.2

[10] S. Al-Ahmad, I. M. Sulaiman, M. Mamat, L. G. Puspa, A Modification of Differential Transform Method for Solving Systems of Second Order Ordinary Differential Equations, Math. Stat., 8 (2020), 464-471. 1 
[11] A. Arikoglu, I. Ozkol, Solutions of integral and integro-differential equation systems by using differential transform method, Comput. Math. Appl., 56 (2008), 2411-2417. 3.1

[12] F. Ayaz, Solutions of the system of differential equations by differential transform method, Appl. Math. Comput., 147 (2004), 547-567. 1

[13] W. G. Belayeh, Y. O. Mussa, A. K. Gizaw, Approximate Analytic Solutions of Two-Dimensional Nonlinear Klein-Gordon Equation by Using the Reduced Differential Transform Method, Math. Probl. Eng., 2020 (2020), 12 pages. 1

[14] B. Benhammouda, H. Vazquez-Leal, A. Sarmiento-Reyes, Modified Reduced Differential Transform Method for Partial Differential-Algebraic Equations, J. Appl. Math., 2014 (2014), 9 pages. 1

[15] J. Biazar, E. Babolian, R. M. Islam, Solution of a system of Volterra integral equations of the first kind by Adomian method, Appl. Math. Comput., 139 (2003), 249-258. 1

[16] J. Biazar, H. Ghazvini, M. Eslami, He's homotopy perturbation method for systems of integro-differential equations, Chaos Solitons Fractals, 39 (2009), 1253-1258. 1

[17] N. Bildik, A. Konuralp, The Use of Variational Iteration Method, Differential Transform Method and Adomian Decomposition Method for Solving Different Types of Nonlinear Partial Differential Equations, Int. J. Nonlinear Sci. Numer. Simul., 7 (2006), 65-70. 1

[18] N. Bildik, A. Konuralp, Two-dimensional differential transform method, Adomian's decomposition method, and variational iteration method for partial differential equations, Int. J. Comput. Math., 83 (2006), 973-987. 1

[19] C.-L. Chen, S.-H. Lin, C.-K. Chen, Application of Taylor transformation to nonlinear predictive control problem, Appl. Math. Modelling, 20 (1996), 699-710. 1

[20] A. T. Deresse, Y. O. Mussa, A. K. Gizaw, Analytical Solution of Two-Dimensional Sine-Gordon Equation, Adv. Math. Phys., 2021 (2021), 15 pages. 1

[21] J.-H. He, Homotopy perturbation technique, Comput. Methods Appl. Mech. Engrg., 178 (1999), 257-262. 1

[22] J.-H. He, A coupling method of a homotopy technique and a perturbation technique for non-linear problems, Internat. J. Non-Linear Mech., 35 (2000), 37-43.

[23] J.-H. He, Homotopy perturbation method: a new nonlinear analytical technique, Appl. Math. Comput., 135 (2003), 73-79.

[24] J.-H. He, Comparison of homotopy perturbation method and homotopy analysis method, Appl. Math. Comput., 156 (2004), 527-539. 1

[25] J.-H. He, Addendum: new interpretation of homotopy perturbation method, Internat. J. Modern Phys. B, 20 (2006), 2561-2568.

[26] J.-H. He, Some asymptotic methods for strongly nonlinear equations, Internat. J. Modern Phys. B, 20 (2006), 1141-1199. 1

[27] B. Ibis, Approximate analytical solutions for nonlinear Emden-Fowler type equations by differential transform method, arXiv, 2012 (2012), 7 pages.

[28] A. F. Jameel, N. R. Anakira, A. K. Alomari, H. M. Noraziah, Solution and Analysis of the Fuzzy Volterra Integral Equations via Homotopy Analysis Method, CMES-Comput. Model. Eng. Sci., 127 (2021), 875-899. 1

[29] A. F. Jameel, N. R. Anakira, M. M. Rashidi, A. K. Alomari, A. Saaban, M. A. Shakhatreh, Differential Transformation Method For Solving High Order Fuzzy Initial Value Problems, Ital. J. Pure Appl. Math., 39 (2018), 194-208. 1

[30] K. Maleknejad, F. Mirzaee, S. Abbasbandy, Solving linear integro-differential equations system by using rationalized Haar functions method, Appl. Math. Comput., 155 (2004), 317-328. 1

[31] S. Momani, V. S. Ertúrk, Solutions of non-linear oscillators by the modified differential transform method, Comput. Math. Appl., 55 (2008), 833-842. 1

[32] S. R. Moosavi Noori, N. Taghizadeh, Modified differential transform method for solving linear and nonlinear pantograph type of differential and Volterra integro-differential equations with proportional delays, Adv. Difference Equ., 2020 (2020), 25 pages. 1

[33] Z. M. Odibat, Differential transform method for solvin Volterra integral equation with separable kernels, Math. Comput. Modelling, 48 (2008), 1144-1149. 1, 2.5

[34] H. Sadeghi Goghary, S. Javadi, E. Babolian, Restarted Adomian method for system of nonlinear Volterra integral equations, Appl. Math. Comput., 161 (2005), 745-751. 1

[35] S.-Q. Wang, J.-H. He, Variational iteration method for solving integro-differential equations, Phys. Lett. A, 367 (2007), 188-191. 1

[36] E. Yusufoglu, An efficient algorithm for solving integro-differential equations system, Appl. Math. Comput., 192 (2007), 51-55. 1

[37] J. K. Zhou, Differential transformation and its application for electrical circuit (in Chinese), Huazhong University Press, Wuuhahn, (1986). 1 